\title{
Comparative study of discretization methods of microarray data for inferring transcriptional regulatory networks
}

\author{
Yong Li ${ }^{1}$, Lili Liu' ${ }^{1}$ Xi Bai ${ }^{1}$, Hua Cai ${ }^{1}$, Wei Ji ${ }^{1}$, Dianjing Guo ${ }^{2^{*}}$, Yanming Zhu ${ }^{1 *}$
}

\begin{abstract}
Background: Microarray data discretization is a basic preprocess for many algorithms of gene regulatory network inference. Some common discretization methods in informatics are used to discretize microarray data. Selection of the discretization method is often arbitrary and no systematic comparison of different discretization has been conducted, in the context of gene regulatory network inference from time series gene expression data.

Results: In this study, we propose a new discretization method "bikmeans", and compare its performance with four other widely-used discretization methods using different datasets, modeling algorithms and number of intervals. Sensitivities, specificities and total accuracies were calculated and statistical analysis was carried out. Bikmeans method always gave high total accuracies.
\end{abstract}

Conclusions: Our results indicate that proper discretization methods can consistently improve gene regulatory network inference independent of network modeling algorithms and datasets. Our new method, bikmeans, resulted in significant better total accuracies than other methods.

\section{Background}

Inferring gene regulatory networks (GRN) using time course microarray data is one of the most important goals in systems biology [1]. A number of algorithms have been proposed to infer the transcription networks, including Boolean Networks [2,3], Gaussian Networks [4], Bayesian Networks [5,6], and Dynamic Bayesian Networks [7]. Most algorithms require discrete data as input. However, the selection of the discretization method is often arbitrary due to the lack of empirical data about the performance of different discretization methods. Discretization methods based on transitions between time points obtain better results than those using absolute values for biclustering time series gene expression data [8]. We proposed therefore that some discretization methods will produce superior results than others when inferring GRN.

\footnotetext{
* Correspondence: djguo@cuhk.edu.hk; Ymzhu2001@neau.edu.cn

${ }^{1}$ Plant Bioengineering Laboratory, Northeast Agricultural University, Harbin,

China

${ }^{2}$ State Key Lab of Agrobiotechnology and Department of Biology, The

Chinese University of Hong Kong, Shatin, N.T., Hong Kong

Full list of author information is available at the end of the article
}

Many discretization methods commonly used in data mining and knowledge discovery have been also used to discretize time series gene expression data (see [8] for review). However, most of these methods are not suitable to be used during preprocessing in time course microarray data analysis, and more specifically they are not suitable, or perform poorly, when used to discretize gene expression data during the process of GRN inference. Discretization algorithms can be divided into two categories: supervised and unsupervised. Supervised methods discretize data with the consideration of class information, but useful class information for inferring GRN is generally not available, so supervised methods are not suitable for inference. Some unsupervised methods, such as "Mid-Ranged", "Max - X\% Max" and "X\% Max" [9], discretize data into only two levels $(0,1)$, so they can not be extensively used for inference.

The purpose of this work was to examine whether there were optimal discretization methods for inferring GRN independent of the network inferring algorithms, number of intervals and datasets. To test this hypothesis, four widely-used and one proposed discretization 
method, "bikmeans", were compared under three network modeling algorithms using different datasets.

\section{Methods}

\section{Discretization methods}

An N-by-M matrix $\mathrm{E}$ is used to denote time course microarray data, where $\mathrm{N}$ is the number of genes, and $\mathrm{M}$ is the number of time points. $E(n, m)$ denotes the expression value of gene $n$ at time point $m$. $E(n,:)$ denotes expression data of gene $n$ at all time points, and $E(:, m)$ denotes expression data of all genes at time point $m$.

\section{(1) Equal Width Discretization (EWD)}

EWD [10-12] divides the number line between $E(n,:)_{\text {min }}$ and $E(n,:)_{\max }$ into $k$ intervals of equal width. Thus the intervals of gene $n$ have width $w=(E(n,:))_{\max }-E(n,:)$ min $) / k$, with cut points at $E(n,:)_{\text {min }}+w, E(n,:)_{\min }+2 w, \cdots$, $E(n,:)_{\min }+(k-1) w . k$ is a positive integer and is a userpredefined parameter.

\section{(2) Equal Frequency Discretization (EFD)}

EFD [10-12] divides the sorted $E(n,:)$ into $k$ intervals so that each interval contains approximately the same number of expression values.

\section{(3) Kmeans Discretization}

Kmeans [13] divides $E(n,:)$ into $k$ intervals by k-means clustering so that adjacent expression values of gene $n$ are divided into same interval.

\section{(4) Column Kmeans Discretization (Cokmeans)}

Cokmeans divides $E(:, m)$ into $k$ intervals by k-means clustering so that adjacent expression values at time point $m$ are divided into same interval.

\section{(5) Bidirectional Kmeans Discretization (Bikmeans)}

Both kmeans and cokmeans are respectively implemented with parameter $k+1$, giving every expression value two discretized values. If the product of the two values is equal to or greater than $x^{2}$, and less than $(x+1)^{2}$, the final discretized value of this expression value is $x$, where $x$ is a positive integer ranging from 1 to $k$. Finally, expression values are divided into $k$ intervals. For example, if one expression value is divided into 3 by kmeans, and 2 by cokmeans with the parameter $k+1=4$, the product is $2 * 3=6$, which is greater than $4\left(=2^{2}\right)$ and

Table 1 A sample of bikmeans discretization method

\begin{tabular}{lllllr}
\hline & & \multicolumn{4}{c}{ Kmeans } \\
\hline \multirow{2}{*}{ Cokmeans } & $\mathbf{1}$ & $\mathbf{2}$ & $\mathbf{3}$ & $\mathbf{4}$ \\
& 1 & 1 & 2 & 3 & 4 \\
& 2 & 2 & 4 & 6 & 8 \\
& 3 & 3 & 6 & 9 & 12 \\
& 4 & 4 & 8 & 12 & 16
\end{tabular}

Kmeans and cokmeans are respectively implemented, firstly. The product of kmeans and cokmeans is used to decide final discretization level. Products $[1-3]$ will be divided into interval $1,[4,6]$ interval 2 and $[8,9,12,16]$ interval 3 . less than $9\left(=(2+1)^{2}\right)$. Therefore, this expression value is divided into the second interval (Table 1).

\section{Microarray data and regulatory networks}

Microarray data and corresponding regulatory networks were generated using ReTRN software [14], which retrieves real yeast microarray data (GEO: GSE4987) [15] and yeast gene regulatory networks http://www.yeastract. $\operatorname{com}[16,17]$. One hundred datasets were generated to compare between the 5 discretization methods. Every dataset contains a 50 -by- 25 (50 genes, 25 time points) time course expression matrix and a corresponding regulatory network. Three network modeling algorithms, namely, Greedy Search, K2 [18] and aracne [19] were used to infer the regulatory network. The parameters used in aracne were $(-\mathrm{p}=1 \mathrm{E}-7,-\mathrm{t}=0.15)$. The parameter "node order" used in $\mathrm{K} 2$ was based on the time points of the initial changes in the time-series expression profiles (up- or down-regulation) of genes. Greater than or equal to 1.2-fold was considered up-regulation and less than or equal to 0.7 -fold was deemed down-regulation as compared to baseline gene expression and these were used as the cutoffs [20]. If the initial change of one gene occurred at an early time point, this gene was selected as potential regulator gene for other genes.

\section{Evaluation of inferred regulatory network}

To evaluate the results of the regulatory network inference, sensitivity (Sn), specificity (Sp) and total accuracy (TA) were calculated for every dataset according to the following equations.

$$
\begin{aligned}
& S n=\frac{T p}{T p+F n} \\
& S p=\frac{T n}{T n+F p} \\
& T A=\frac{T n+T p}{T n+F n+T p+F p}
\end{aligned}
$$

$\mathrm{Tp}$ (true positive) is the number of regulatory relations correctly inferred. Tn (true negative) is the number of nonregulatory relations correctly inferred. Fn (false negative) is the number of regulatory relations incorrectly inferred as non-regulatory relations. Fp (false positive) is the number of non-regulatory relations incorrectly inferred as regulatory relations. TA is a synthetic index for evaluation.

\section{Results}

Using the ReTRN software, 100 datasets were generated to infer GRNs using five discretization methods, three interval levels and three network modeling algorithms. 


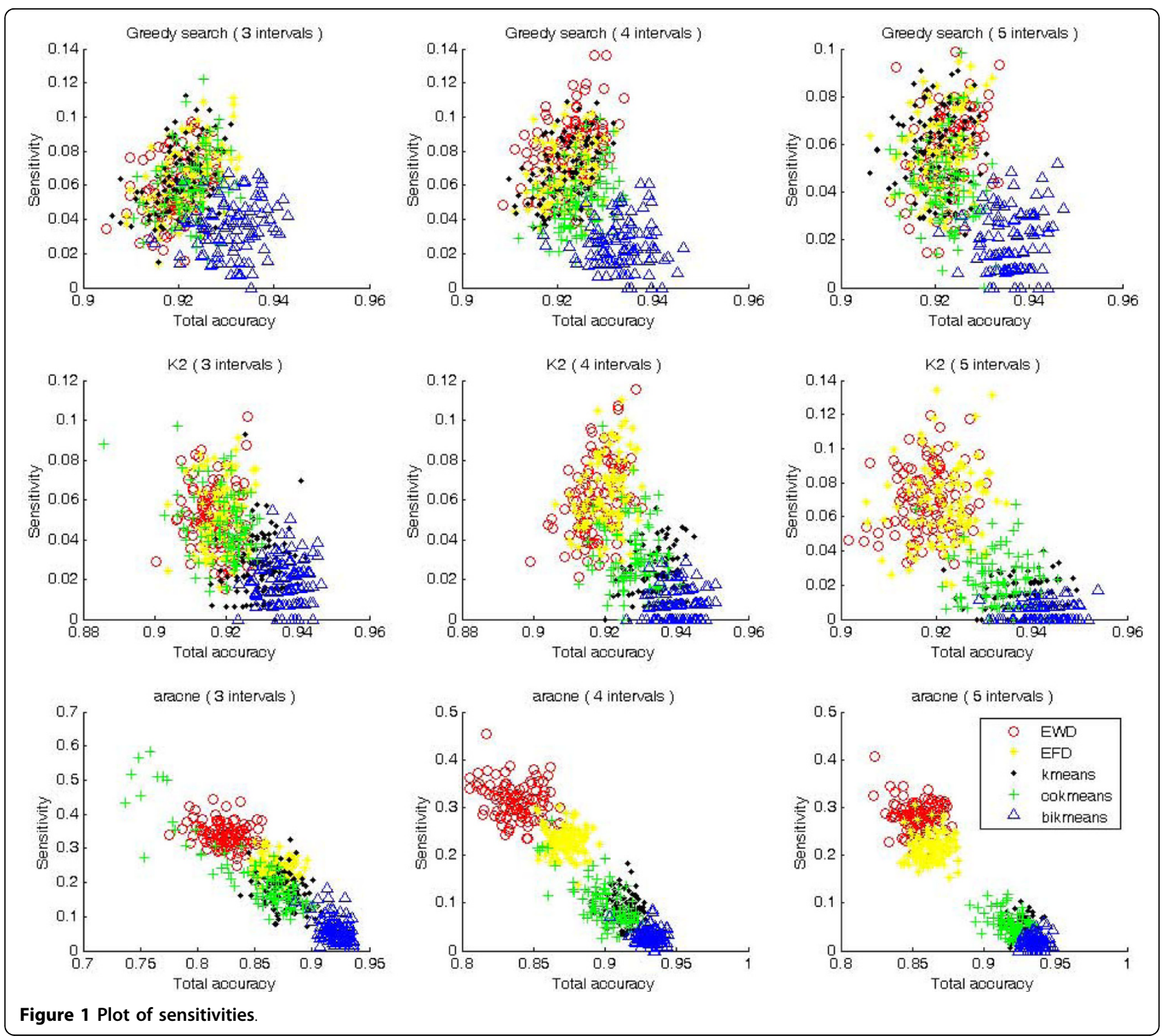

Inferred networks were then compared with real regulatory networks to calculate sensitivity, specificity, and total accuracy (Figures 1, 2).

As shown in Figures 1 and 2, every discretization method was distributed on a successive field, indicating that every discretization method results in similar sensitivities, specificities, and total accuracies, even though different datasets were used. Bikmeans was easily distinguishable from other methods because it produced much higher total accuracies under all situations. In general, bikmeans had relatively low sensitivities (Figure 1 ), but high specificities (Figure 2), which collectively produced high total accuracies. This indicates that most regulatory relations found by bikmeans are correct.

Three-way analysis of variance revealed that total accuracies of five discretization methods were significantly different, irrespective of inferring algorithms and number of intervals (Table 2). Every factor (inferring algorithm, discretization method and number of intervals) and combinations of the factors significantly influence total accuracy. The inferring algorithm had the biggest effect on total accuracy, followed by the discretization method. The number of intervals had the least effect on total accuracy. Multiple comparisons (Figure 3) revealed more details on the effect of combinations of factors. Eight of the 12 combinations which significantly improved total accuracies utilized the bikmeans method.

\section{Discussion}

In this paper, we compared and contrasted several widely-used discretization methods for inferring GRN 


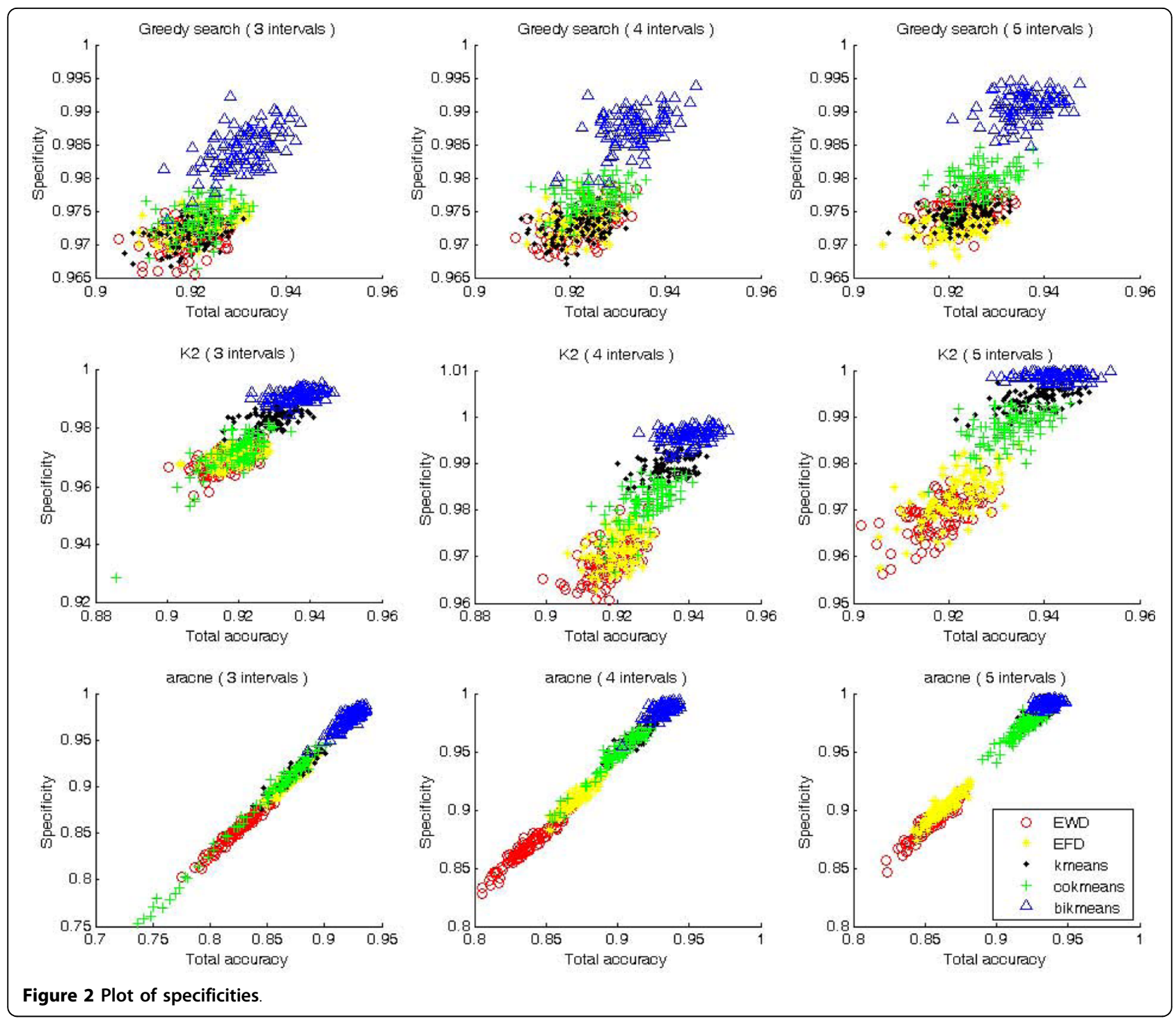

Table 2 Three-way analysis of variance of total accuracy

\begin{tabular}{cccccc}
\hline Source & Sum Sq. & d.f. & Mean Sq. & $\mathbf{F}$ & $\mathbf{P}$ \\
\hline S1 & 1.569 & 2 & 0.7843 & 6845.56 & 0 \\
S2 & 0.147 & 2 & 0.0735 & 641.56 & 0 \\
S3 & 0.922 & 4 & 0.2306 & 2013.03 & 0 \\
S1 ${ }^{*}$ S2 & 0.128 & 4 & 0.0320 & 279.38 & 0 \\
S1 ${ }^{*}$ S3 & 0.683 & 8 & 0.0854 & 745.49 & 0 \\
S2 ${ }^{\text {S3 }}$ & 0.080 & 8 & 0.0100 & 87.67 & 0 \\
Error & 0.512 & 4471 & 0.0001 & & \\
Total & 4.042 & 4499 & & &
\end{tabular}

S1: Inferring algorithm.

S2: Number of intervals.

S3: Discretization method.

Sum Sq.: sum of squares.

d.f.: degrees of freedom.

Mean Sq.: mean squares, the ratio Sum Sq./d.f.

F: F-statistic.

$\mathrm{P}$ : $\mathrm{p}$-value, derived from the Cumulative Distribution Function (cdf) of $\mathrm{F}$. with our proposed new method and found that discretization methods gave consistent performance independent of the network inferring algorithms, number of intervals and datasets used. Bikmeans method resulted in a greater number of correct inferred results, even when using the arcane algorithm, which generally yielded relatively low total accuracies. This result suggests that bikmeans is the most suitable discretization method for inferring GRN.

EWD and EFD are sensitive to extreme and arbitrary values. Kmeans clusters adjacent values from the same row or column into the same interval, and discretized values can better reflect the real information. Row kmeans discretizes row expression values at all time points, representing a gene profile, and column kmeans discretizes column expression values at one time point, generally representing a microarray chip. To infer GRN, reducing 


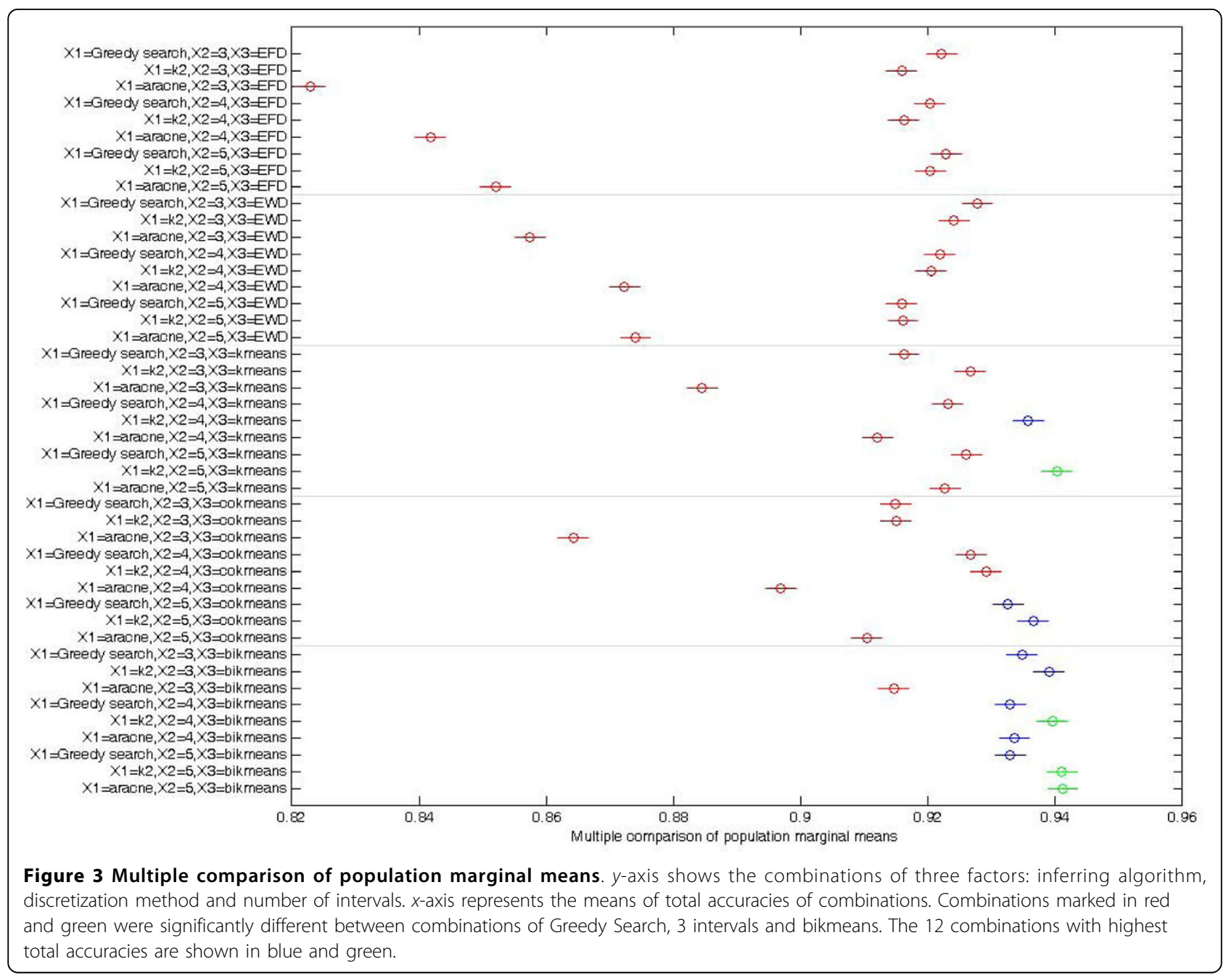

dimensions by excluding unrelated genes from microarray is a necessary preprocess [22], so these genes which are selected to infer GRN have potential regulatory relations. Among these genes, some may have small expression change range, but they function as regulators in the regulatory process. Transcription factor and microRNA (miRNA) genes are examples of these regulators, so their expression values should be discretized into same number of intervals, which can be achieved by row kmeans. To keep gene regulatory information in a microarray chip, column expression values should be discretized into different intervals, which can be achieved by column kmeans. According to the algorithms, if an expression value is very high among its row, and low among its column, row kmeans would discretize this value into high interval, and column kmeans would polish it. So bikmeans is a compatible method that implements kmeans at the row and column, and then combines the two results. This method reflects expression changes within and between genes, which is what inferring algorithms that discover regulatory relations are based on. Therefore, as expected, bikmeans had greater total accuracies, making it most suitable discretization method for inferring GRN. Of course, it may be also suitable for other aspects, such as clustering and classification, which are not analyzed in this study.

\section{Conclusions}

Choosing a correct discretization method can improve the accuracy of inferring GRN, but is it independent of the network inferring algorithms and datasets? How much it influences accuracy? Based on the results from this study, we conclude that it is critical in improving the accuracy of GRN inference, and good discretization method result in higher accuracies independent of the network inferring algorithms, number of intervals and datasets used, but the inferring algorithm has the bigger effect on total accuracy than discretization method. In addition, our new bikmeans method, designed according to the mechanism of inferring GRN, obtained better results than other methods with typical data sets. 


\section{Abbreviations}

GRN: Gene Regulatory Network; EWD: Equal Width Discretization; EFD: Equal Frequency Discretization; Cokmeans: Column kmeans discretization; Bikmeans: Bidirectional kmeans discretization; Sn: Sensitivity; Sp: Specificity; Tn: True negative; Tp: True positive; Fn: False negative; Fp: False positive; TA: Total Accuracy.

\section{Acknowledgements}

This project was supported by a grant from the National Natural Science Foundation of China (30570990), the Hong Kong UGC AoE Plant \& Agricultural Biotechnology Project AoE-B-07/09 and the Institute of Plant Molecular Biology and Agrobiotechnology at The Chinese University of Hong Kong.

\section{Author details}

'Plant Bioengineering Laboratory, Northeast Agricultural University, Harbin, China. ${ }^{2}$ State Key Lab of Agrobiotechnology and Department of Biology, The Chinese University of Hong Kong, Shatin, N.T., Hong Kong.

\section{Authors' contributions}

$Y L$ designed the study, participated in its implement and coordination, and drafted the manuscript. LLL participated in its design, and carried out the statistical analysis. XB, HC and WJ helped with statistical analysis. DJG and YMZ participated in its design and coordination, and helped with the manuscript editing. All authors read and approved the final manuscript.

Received: 27 June 2010 Accepted: 19 October 2010 Published: 19 October 2010

\section{References}

1. Laubenbacher R, Stigler B: A computational algebra approach to the reverse engineering of gene regulatory networks. J Theor Biol 2004, 229(4):523-537.

2. Somogyi R, Sniegoski C: Modeling the complexity of genetic networks: Understanding multigenic and pleiotropic regulation. Complexity 1996, 1:45-63.

3. Akutsu T, Miyano S, Kuhara S: Identification of genetic networks from a small number of gene expression patterns under the Boolean network model. Pac Symp Biocomput 1999, 17-28.

4. Wille A, Zimmermann P, Vranova E, Furholz A, Laule O, Bleuler S, Hennig L, Prelic A, von Rohr $P$, Thiele $L$, et al: Sparse graphical Gaussian modeling of the isoprenoid gene network in Arabidopsis thaliana. Genome Biol 2004, 5(11):R92.

5. Friedman $N$, Linial M, Nachman I, Pe'er D: Using Bayesian networks to analyze expression data. J Comput Biol 2000, 7(3-4):601-620.

6. Hartemink AJ, Gifford DK, Jaakkola TS, Young RA: Combining location and expression data for principled discovery of genetic regulatory network models. Pac Symp Biocomput 2002, 437-449.

7. Murphy K, Mian S: Modeling gene expression data using dynamic Bayesian networks. Technical report. Computer Science Division, University of California, Berkeley, CA 1999 [http://www.cs.ubc.ca/ murphyk/Papers/ ismb99.pdf].

8. Madeira SC, Teixeira MC, Sa-Correia I, Oliveira AL: Identification of Regulatory Modules in Time Series Gene Expression Data Using a Linear Time Biclustering Algorithm. IEEE/ACM Trans Comput Biol Bioinformatics 2010, 7(1):153-165.

9. Pensa R, Leschi C, Besson J, Boulicaut JF: Assessment of discretization techniques for relevant pattern discovery from gene expression data. 4th ACM SIGKDD Workshop on Data Mining in Bioinformatics 2004, 24-30, BIOKDD'04, ACM.

10. Catlett J: On changing continuous attributes into ordered discrete attributes. Proceedings of the European working session on learning on Machine learning Porto, Portugal: Springer-Verlag New York, Inc 1991, 164-178.

11. Dougherty J, Kohavi R, Sahami M: Supervised and Unsupervised Discretization of Continuous Features. Proceedings of the Twelfth International Conference on Machine Learning: 1995; Tahoe City, California, USA 1995, 194-202.

12. Randy K: Chimerge: discretization of numeric attributes. Proceedings Tenth National Conference on Artificial Intelligence Publ by AAAl, Menlo Park, CA, United States 1992.
13. MacQueen JB: Some Methods for Classification and Analysis of MultiVariate Observations. In Proc of the fifth Berkeley Symposium on Mathematical Statistics and Probability. Edited by: Cam LML, Neyman J. University of California Press; 1967:1:281-297.

14. Li Y, Zhu Y, Bai X, Cai H, Ji W, Guo D: ReTRN: A retriever of real transcriptional regulatory network and expression data for evaluating structure learning algorithm. Genomics 2009, 94(5):349-354.

15. Pramila T, Wu W, Miles S, Noble WS, Breeden LL: The Forkhead transcription factor $\mathrm{Hcm} 1$ regulates chromosome segregation genes and fills the S-phase gap in the transcriptional circuitry of the cell cycle. Genes Dev 2006, 20(16):2266-2278.

16. Monteiro PT, Mendes ND, Teixeira MC, d'Orey S, Tenreiro S, Mira NP, Pais H, Francisco AP, Carvalho AM, Lourenco AB, et al: YEASTRACT-DISCOVERER: new tools to improve the analysis of transcriptional regulatory associations in Saccharomyces cerevisiae. Nucleic Acids Res 2008, , 36 Database: D132-136.

17. Teixeira MC, Monteiro $P$, Jain $P$, Tenreiro $S$, Fernandes AR, Mira NP, Alenquer M, Freitas AT, Oliveira AL, Sa-Correia I: The YEASTRACT database: a tool for the analysis of transcription regulatory associations in Saccharomyces cerevisiae. Nucleic Acids Res 2006, , 34 Database: D446-451

18. Cooper GF, Herskovits E: A Bayesian method for the induction of probabilistic networks from data. Machine Learning 1992, 9(4):309-347.

19. Margolin AA, Nemenman I, Basso K, Wiggins C, Stolovitzky G, Dalla Favera R, Califano A: ARACNE: an algorithm for the reconstruction of gene regulatory networks in a mammalian cellular context. $B M C$ Bioinformatics 2006, 7(Suppl 1):S7.

20. Zou M, Conzen SD: A new dynamic Bayesian network (DBN) approach for identifying gene regulatory networks from time course microarray data. Bioinformatics 2005, 21(1):71-79.

21. Kurgan LA, Cios KJ: CAIM Discretization Algorithm. IEEE Trans on Knowl and Data Eng 2004, 16(2):145-153.

22. Xu R, Wunsch li $D$, Frank R: Inference of genetic regulatory networks with recurrent neural network models using particle swarm optimization. IEEE/ACM Trans Comput Biol Bioinform 2007, 4(4):681-692.

doi:10.1186/1471-2105-11-520

Cite this article as: Li et al:: Comparative study of discretization methods of microarray data for inferring transcriptional regulatory networks. BMC Bioinformatics 2010 11:520.

\section{Submit your next manuscript to BioMed Central and take full advantage of:}

- Convenient online submission

- Thorough peer review

- No space constraints or color figure charges

- Immediate publication on acceptance

- Inclusion in PubMed, CAS, Scopus and Google Scholar

- Research which is freely available for redistribution

Submit your manuscript at www.biomedcentral.com/submit
C Biomed Central 